\title{
Distribución y caracterización del daño ocasionado por Pissodes castaneus en plantaciones de Pinus spp. de la región Andino Patagónica Argentina
}

\author{
Distribution and characterization of damage caused by Pissodes castaneus \\ in Pinus spp. plantations of the Andean Patagonian Region, Argentina \\ Cecilia Gomez $^{\text {a*}, ~ N a t a l i a ~ C ~ V a l l e j o s ~}{ }^{\text {a }}$, Ludmila La Manna a,b \\ *Autor de correspondencia: ${ }^{a}$ Universidad Nacional de la Patagonia San Juan Bosco, Facultad de Ingeniería, \\ Ruta 259 km 16,4, Esquel (CP 9200), Chubut, Argentina, tel.: 02945-450820, ceciligomez@gmail.com \\ ${ }^{\mathrm{b}}$ Consejo Nacional de Investigaciones Científicas y Técnicas, Buenos Aires, Argentina.
}

\begin{abstract}
SUMMARY
Pissodes castaneus (Col.: Curculionidae) was first reported in the Patagonian Andean region in Neuquén province in 2005. This weevil generally attacks trees previously affected by other biotic or abiotic factors. In some plantations it is the main sanitary problem; however, its distributional area and infestation levels are still unknown. The aims of this study were: 1) to assess the current pest distribution in the Patagonian Andean Region, 2) to characterize and quantify the damage caused by Pissodes castaneus in Chubut province, and 3) to evaluate the association between infestation levels and silvicultural and environmental conditions in Chubut province plantations. Pissodes castaneus was registered in Neuquén, Río Negro and Chubut provinces, being the proportion of infested plantations higher in the last provinces than in Neuquén. Infestation level ranged between $1.4 \%$ and $45.7 \%$. Pinus contorta var. murrayana was the most affected species followed by Pinus radiata and Pinus ponderosa. The major mortality was registered in Pinus contorta var. murrayana. However, in young plantations of Pinus ponderosa it was found only as a damage agent causing the death of trees by annealing. Infestation level was positively associated to woody debris and to Sirex noctilio attack. Characteristics of the attack and the distribution reached by the insect in the region suggest that Pissodes castaneus should be considered in management guidelines.
\end{abstract}

Key words: infestation level, distribution, damages, silvicultural management.

\section{RESUMEN}

El primer registro de Pissodes castaneus (Col.: Curculionidae) en la región Andino Patagónica fue realizado en 2005 en la provincia de Neuquén. Este insecto ataca coníferas del género Pinus, generalmente debilitadas por factores bióticos o abióticos. En algunas plantaciones constituye el único problema sanitario, sin embargo, se desconoce su área de distribución y los niveles de daño en la región. Los objetivos del trabajo fueron: 1) determinar la distribución actual de la especie en la región Andino Patagónica, 2) tipificar y cuantificar el daño ocasionado por Pissodes castaneus en plantaciones de la provincia de Chubut, y 3) evaluar la asociación entre intensidad de ataque y características silvícolas y ambientales de las plantaciones en la provincia de Chubut. La especie fue registrada en las provincias de Neuquén, Río Negro y Chubut, siendo proporcionalmente mayor el número de plantaciones infestadas en las dos últimas. Los porcentajes de infestación variaron entre 1,4 y 45,7 \%. Pinus contorta var. murrayana resultó más afectado y, en orden de importancia, le siguieron Pinus radiata y Pinus ponderosa. La mayor mortalidad se registró en Pinus contorta var. murrayana. En plantaciones jóvenes de Pinus ponderosa fue registrado como el único agente de daño, ocasionando la muerte por anillamiento. El grado de infestación estuvo positivamente asociado con la presencia de residuos forestales y con el ataque de Sirex noctilio. Las características del ataque observado en la región, sumadas a la distribución que ha alcanzado la especie, exigen que Pissodes castaneus sea considerado en la definición de pautas de manejo.

Palabras clave: nivel de infestación, distribución, daños, manejo silvicultural.

\section{INTRODUCCIÓN}

En la región Andino Patagónica Argentina las plantaciones de coníferas exóticas del género Pinus ocupan una superficie de 100.000 ha (MINAGRI 2012). Las principales especies utilizadas son Pinus ponderosa Dougl. ex Laws., Pinus contorta Dougl. var. murrayana y Pinus radiata D. Don.
Sirex noctilio Fabricius (Hym.: Siricidae) es el principal agente de daño que afecta la sanidad de estos bosques. Esta plaga primaria introducida a principios de la década del 90 en la región, constituye además el mayor problema sanitario en Pinus spp. en Argentina (Villacide y Corley 2012). Aparte de ocasionar importantes pérdidas económicas por la muerte de los árboles disminuye el valor escé- 
nico-paisajístico de las masas forestales en la Patagonia Andina. En los últimos años se ha sumado el daño que ocasiona Pissodes castaneus De Geer (Col.: Curculionidae) (Gomez y Hartel 2010). Este insecto también es originario de Europa y Norte de África, donde se distribuye ampliamente e incluso está presente en Siberia (Bichão et al. 2003) y Turquía (Panzavolta 2007). En América del Sur $P$. castaneus se introdujo y estableció en Uruguay (Morey y Porcile 1992) y Brasil (Ribeiro 2009) y constituye una especie cuarentenaria para Chile (SAG 2013). En Argentina se tienen registros de $P$. castaneus desde fines de la década del 90, donde se detectaron daños ocasionados por la especie en el norte y centro del país (Lanteri et al. 2002). No obstante, la presencia de la especie en Argentina dataría desde 1947. La revisión de material depositado en el Museo de La Plata (División Entomología), Buenos Aires, Argentina, reportó la existencia de una serie de tres ejemplares identificados como Pissodes notatus colectados sobre Pinus sp., en el partido de Magdalena (provincia de Buenos Aires). En la Región Andino Patagónica $P$. castaneus fue registrado y reportado formalmente en 2005, en plantaciones de Pinus contorta var. murrayana en Neuquén (Fontana et al. 2006). En tanto que en Chubut en 2006, se encontró la plaga afectando forestaciones situadas en el noroeste de la provincia, Cholila, El Maitén y Esquel (Gomez et al. 2011). Por otro lado, también está presente en la provincia de Río Negro desde hace algunos años (Gomez et al. 2011).

Las larvas se alimentan del cambium y floema, tanto en fustes como ramas, e incluso alcanzan las raíces. Los adultos horadan pequeños orificios en la corteza para alimentarse; los que luego son rápidamente cubiertos por cicatrices de tejido (Schwenke 1974, Panzavolta y Tiberi 2010). En su área de distribución natural se comporta, en general, como un insecto secundario asociado al ataque previo de otros agentes de daño (Schwenke 1974), infestando individuos debilitados por alguna condición previa (Romanyk y Cadahia 2003) o bien individuos recientemente cortados (Day et al. 2004). En Alemania es frecuente su aparición en pinos jóvenes, previamente atacados por insectos descortezadores y sus estallidos poblacionales están relacionados con plantaciones debilitadas por el ataque de barrenadores de yemas y brotes del género Rhyacionia (Lep.: Tortricidae), así como también rodales más viejos afectados previamente por el defoliador Diprion pini L. (Schwenke 1974) (Hym.: Diprionidae). Por otro lado, las plantaciones debilitadas por condiciones climáticas o edáficas estresantes, o por un inadecuado manejo silvicultural también son más propensas al ataque. En este sentido, las plantaciones jóvenes situadas en sitios pobres como suelos poco profundos o inundables o sometidos a la sequía, o bien cuyas características no responden a la especie forestal empleada, son muy susceptibles. Además, es frecuente la aparición de focos de $P$. castaneus en rodales afectados por incendios (Romanyk y Cadahia 2003). No obstante, también existen observaciones de Pissodes spp. afectan- do árboles aparentemente sanos $-P$. castaneus entre otras especies- especialmente cuando alcanza altas densidades de población (Kudela 1974, Day et al. 2004). En Brasil, donde es una especie introducida, su presencia está estrechamente relacionada con la condición silvicultural de la plantación (e.g. calidad del sitio, condición física del suelo, técnica de plantación, deficiencia nutricional) y la ocurrencia de fenómenos abióticos (e.g. granizo, heladas, sequía prolongada, entre otros). Los estallidos poblacionales pueden comenzar en plantaciones jóvenes, afectando usualmente pocos árboles, pero luego la población puede crecer rápidamente. El incremento inicial de la población del insecto se asocia con la existencia de un gran número de árboles susceptibles (Iede et al. 2010).

Respecto del rango de hospederos y sus preferencias, todas las especies europeas de Pissodes están restringidas mayoritariamente al género Pinus, mientras que algunas especies norteamericanas como Pissodes strobi Peck o Pissodes nemorensis Germar atacan comúnmente especies de Pinus y Picea (O'Brien 1989). Sin embargo, también existen algunos reportes para $P$. castaneus sobre Abies sp. y Larix sp. (Day et al. 2004). Pissodes castaneus se asocia con casi todas las especies del género Pinus, dentro de su rango de distribución natural, y prefiere árboles jóvenes de entre 4 y 15 años. A pesar de ello se observan diferencias significativas en su éxito reproductivo y desarrollo según la especie hospedera donde se haya alimentado y criado (Day et al. 2004).

En la región Andino Patagónica Argentina la situación sanitaria de las forestaciones respecto de esta especie pareciera haberse agravado desde su detección (Gomez y Hartel 2010). Desde el reporte formal de P. castaneus en Chubut en 2006, se ha observado una amplia distribución de la especie en forestaciones de Pinus spp. bajo diferentes condiciones silvícolas y ambientales; incluso, en plantaciones de Pinus ponderosa bajo manejo, que es la especie que se planta mayormente en la región. Este hecho sugiere que el gorgojo estaría presente en niveles bajos de prevalencia, desde hace algunos años (Gomez y Hartel 2010).

El conocimiento de esta especie en la región aún es limitado, en especial sobre aquellos aspectos relacionados con su biología y ecología, como así también la situación sanitaria de las forestaciones. En este sentido, y como parte del manejo efectivo de una plaga, es necesario conocer su distribución y abundancia, especialmente en relación con la disponibilidad de material de cría. El monitoreo, es por lo tanto, un elemento importante en la toma de decisiones sobre si se deben aplicar medidas activas de control.

A fin de aportar al conocimiento de la especie en la región, se plantearon los siguientes objetivos: 1) determinar la distribución actual de la especie en la región Andino Patagónica, 2) tipificar y cuantificar el daño ocasionado por $P$. castaneus en plantaciones de la provincia de Chubut, y 3) evaluar la asociación entre la intensidad del ataque y las principales características silvícolas y ambientales de las plantaciones de la provincia de Chubut, bajo la hipótesis 
que en plantaciones debilitadas por mal manejo silvícola o por presencia de otras plagas, es mayor la intensidad de ataque por $P$. castaneus.

\section{MÉTODOS}

Distribución de la plaga en la región Andino Patagónica. Para conocer la distribución de la plaga en la región, se muestrearon 61 plantaciones durante la primavera-verano de 2011-2012. En estas plantaciones, ubicadas en las provincias de Chubut, Río Negro y Neuquén (figura 1), se registró presencia/ausencia a través del reconocimiento de los signos, síntomas y daños característicos del ataque. Se efectuaron recorridos aleatorios en distintos rodales de la plantación visitada para la inspección visual e identificación de la sintomatología característica. Para constatar la presencia del insecto en los sitios donde no se pudo confirmar fehacientemente el ataque, por ejemplo, en rodales con árboles de altura elevada que dificultaban su observación, se voltearon los árboles "presumiblemente" atacados y se descortezaron parcialmente in situ en busca de galerías, cámaras pupales bajo la corteza u otro signo de ataque del insecto. Asimismo, de estos árboles se cortaron trozas de $80 \mathrm{~cm}$ de longitud; una parte de estas trozas fue conservada en cámaras de crianza a la espera de la emergencia de adultos, mientras que otra parte de las mismas se procesó en el laboratorio, especialmente, cuando en el campo no se evidenciaron galerías en las plantas volteadas.

Caracterización del daño y determinación del nivel de infestación en la provincia de Chubut. En las 35 plantaciones ubicadas en la provincia de Chubut y en el límite

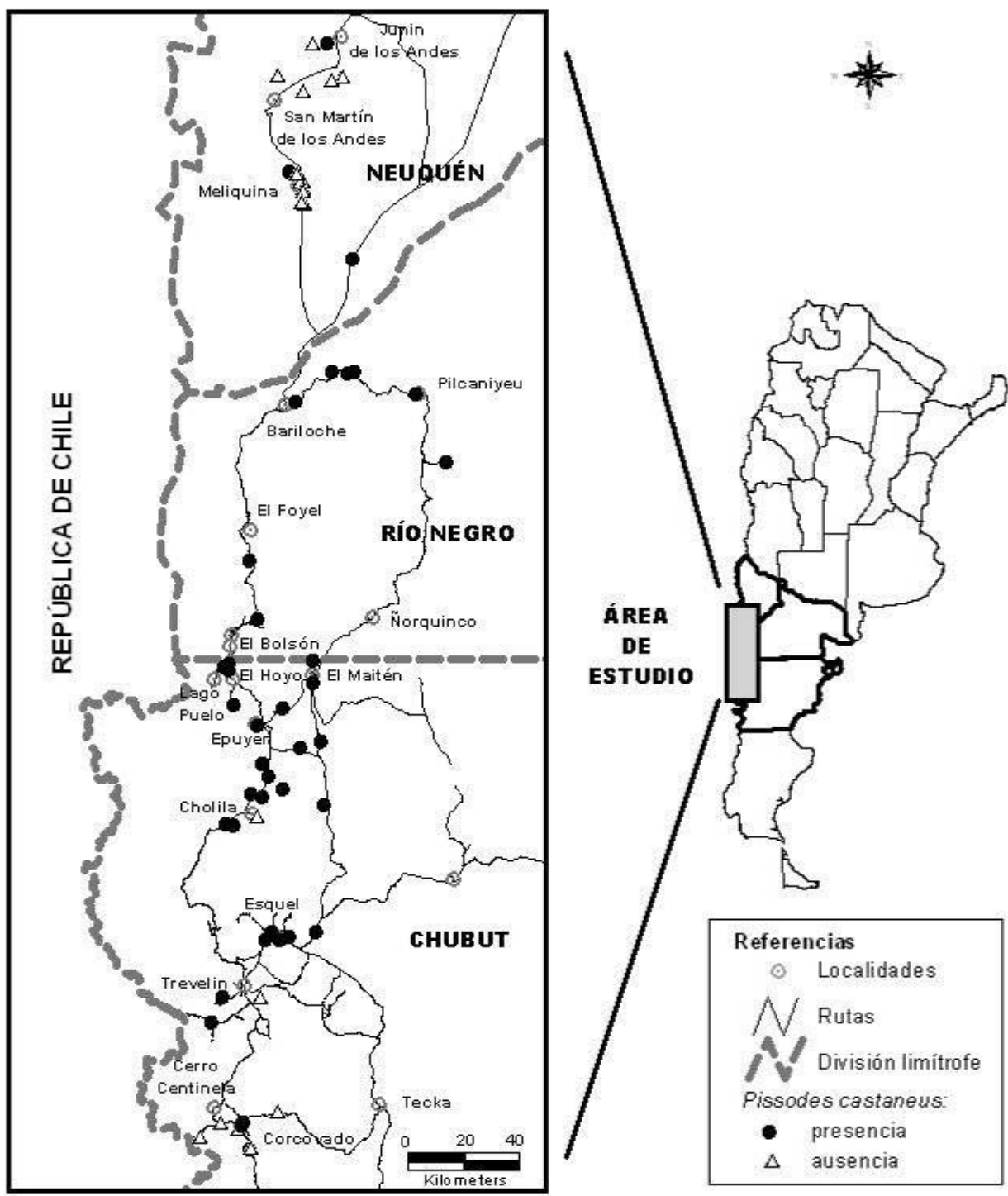

Figura 1. Área de estudio y distribución de las plantaciones evaluadas. Study area and location of the sampled plantations. 
Chubut-Río Negro (figura 1) se realizó la cuantificación del daño. Se tomaron como criterios de selección de los sitios a muestrear: el gradiente de precipitación este-oeste característico de la región, especies forestales, edad de las plantaciones, situación de manejo silvicultural y situación fitosanitaria en relación con S. noctilio, tomando como base las prospecciones y monitoreos que lleva adelante la Subsecretaría de Bosques de Chubut para el manejo de S. noctilio. La representatividad de las distintas especies forestales muestreadas estuvo limitada por las existencias en la región, siendo Pinus ponderosa la especie más plantada y promocionada debido a su plasticidad.

Para evaluar el nivel de infestación por $P$. castaneus se procedió a muestrear el $5 \%$ de los individuos de la plantación, distribuidos en transectos orientados según la dirección de las líneas de plantación. A partir de la cobertura digital de los polígonos de cada plantación, se seleccionaron al azar puntos sobre el área a muestrear, los que constituyeron las coordenadas de ubicación del inicio de cada transecto. En aquellas forestaciones con un área menor o igual a 10 ha, se evaluó cada tres individuos la presencia o no de ataque de $P$. castaneus, observando un total de 50 árboles por transecto. En las plantaciones con superficie mayor a 10 ha, la presencia de la plaga fue evaluada cada cinco plantas a lo largo de cada transecto, considerando un total de 100 plantas por transecto. En aquellos casos en que la línea de plantación finalizó antes de haber muestreado la totalidad de los individuos previstos, se continuó observando la línea contigua.

Se identificaron y sistematizaron los diferentes tipos de síntomas o daños que se relacionan con distintos grados de avance del ataque: transparencia de la copa, decoloración del follaje, defoliación avanzada, resquebrajamiento y descortezamiento de la corteza, ubicación del ataque a lo largo del fuste o ramas, coloración y humedad de las fibras que protegen las cámaras pupales para determinar si corresponden a la temporada de emergencia actual, o a temporadas previas en las que no ocurrió la emergencia del adulto.

En cada árbol muestreado se registró la presencia/ausencia de ataque de $P$. castaneus, la especie forestal, su posición relativa dentro del dosel y la tipificación sintomática expuesta anteriormente, además de la coexistencia de otra plaga en la misma planta.

Asociación entre la intensidad del ataque y las condiciones silvícolas y ambientales. Para cada plantación se determinó su ubicación geográfica mediante un sistema de posicionamiento global (GPS), se realizó su caracterización dasométrica y silvicultural, y se evaluó la situación sanitaria respecto de S. noctilio. La caracterización dasométrica de cada plantación se efectúo utilizando una parcela circular de $300 \mathrm{~m}^{2}$, elegida al azar. En cada parcela se midió el diámetro a la altura del pecho (DAP $=1,30 \mathrm{~m}$ ) de todos los árboles incluidos en la parcela y la altura de los tres árboles de mayor DAP (Assmann 1970). Se determinó el diámetro cuadrático medio (DCM) y el índice de densidad de Reineke (IDR) (Daniel et al. 1982). La edad de la plantación se tomó de los registros preexistentes en la Subsecretaría de Bosques o a través de la consulta a sus propietarios. En aquellas situaciones en las que no se pudo disponer del registro, se contó el número de entrenudos de los tres árboles dominantes de la parcela para Pinus ponderosa (Gonda 2001), mientras que para Pinus radiata y Pinus contorta var. murrayana se realizó el conteo del número de anillos. La edad máxima de las plantaciones muestreadas fue 40 años para las distintas especies. La edad mínima en cambio fue 12 años para Pinus ponderosa y Pinus radiata y 20 años para Pinus contorta var. murrayana. Las variables silviculturales relevadas fueron la existencia y tipo de tratamiento practicado (podas y raleos) y manejo de los residuos. El grado de ataque de S. noctilio se determinó mediante el muestreo secuencial que es la metodología habitual utilizada en la región e implementada actualmente por la Subsecretaría de Bosques.

Se evaluó la correlación entre el porcentaje de ataque por $P$. castaneus y las distintas variables de sitio relevadas, incluyendo la latitud, longitud, edad de la plantación, porcentaje de infestación por $S$. noctilio, diámetro cuadrático medio, índice de densidad de Reineke y presencia de residuos forestales (variable dicotómica). Para cada variable, se calcularon los coeficientes de correlación de Pearson o Spearman, de acuerdo al cumplimiento o no de los supuestos de normalidad y homocedácea. Se realizó también un análisis de componentes principales (ACP), a fin de evaluar las variables de sitio en conjunto. El análisis de componentes principales incluyó las variables latitud, edad de la plantación, porcentaje de infestación por $S$. noctilio, índice de densidad de Reineke y presencia de residuos forestales. Luego, se analizó la correlación entre los ejes del análisis de componentes principales y el porcentaje de infestación por $P$. castaneus. Los análisis estadísticos fueron realizados con el programa Infostat.

\section{RESULTADOS}

Distribución de la plaga en la región Andino Patagónica. Pissodes castaneus estuvo presente en las tres provincias de la región Andino Patagónica, siendo variable su frecuencia de aparición en cada una de ellas.

En Neuquén el número de plantaciones infestadas resultó proporcionalmente menor respecto de Río Negro y Chubut. En esta provincia se relevaron 18 plantaciones situadas al sur, de las cuales en sólo tres se registró la presencia de $P$. castaneus (figura 1). Únicamente en la plantación situada más al este, en condiciones de baja calidad de sitio, se observó el daño en árboles vivos y muertos en pie, donde se encontraron orificios de emergencia, cámaras pupales y larvas vivas en fustes de Pinus contorta var. murrayana. En las restantes forestaciones afectadas - de la especie Pinus ponderosa - la presencia de $P$. castaneus se constató sólo en los residuos forestales, donde se observaron cámaras pupales, y no se registraron árboles afectados en pie. 
En las ocho plantaciones visitadas en la provincia de Río Negro, se registró la presencia de $P$. castaneus mediante los síntomas y signos típicos, claramente reconocibles tanto en las plantas en pie como en los residuos forestales. La mayor parte de estas forestaciones correspondieron a Pinus contorta var. murrayana, en menor medida a Pinus ponderosa o bien a forestaciones mixtas de ambas especies.

En la provincia de Chubut se relevaron 35 plantaciones, registrándose la presencia de $P$. castaneus en 27 de ellas.

Caracterización del daño y determinación del nivel de infestación en la provincia de Chubut. El ataque de P. castaneus se observó situado en el ápice o generalizado en toda la planta, dependiendo de la especie forestal, altura de la planta y presencia/ausencia de otras plagas. En árboles jóvenes (12 a 16 años) y de alturas entre 3 y 6 m se observó la muerte por anillamiento, mientras que en plantas adultas y de mayor altura (20 a 29 m) sólo se observó la muerte apical, quedando concentrado el ataque en esa área.

En el sur de la provincia la sintomatología observada correspondió a la de un ataque incipiente. En Corcovado la presencia de la plaga sería relativamente reciente y aún no alcanza niveles de infestación que permitan la detección visual clara. Por otra parte, en la zona centro y norte de Chubut la sintomatología observada correspondió con un ataque de mayor antigüedad. En estos casos, la sintomatología puede resumirse en la siguiente secuencia:

a. Transparencia de la copa por defoliación apical incipiente acompañada de hinchazón y agrietamiento de la corteza en forma de resquebrajaduras transversales.

b. Desprendimiento de la corteza acompañada de un aumento de defoliación.

c. Decoloración marcada del follaje desde el ápice.

d. Muerte de ápices y ramas (figura 2).

e. Muerte de la planta completa, en general con cámaras pupales hasta la base de la planta e incluso en las raíces.

Los porcentajes de infestación variaron entre un mínimo de $1,4 \%$ y un máximo de $45,7 \%$. El mayor valor correspondió a una forestación afectada por el fuego situada en el límite entre las provincias de Chubut y Río Negro $\left(41^{\circ}\right.$ 52’32,6” S), mientras que el mínimo ataque correspondió a una plantación situada en la zona sur del área de muestreo (43 32'39,7” S), cercana a la localidad de Corcovado.

Cinco de las siete plantaciones en las que $P$. castaneus estuvo ausente se ubicaron hacia el sudoeste de la provincia, próximas a la localidad de Corcovado, prácticamente, hacia el límite sur de la zona forestada en la región cordillerana (figura 1).

Asociación entre la intensidad del ataque y las condiciones silvícolas y ambientales. Existió una correlación negativa entre el grado de infestación por $P$. castaneus y la latitud ( $\mathrm{r}=-0,53 ; P=0,001)$, siendo mayor el grado de

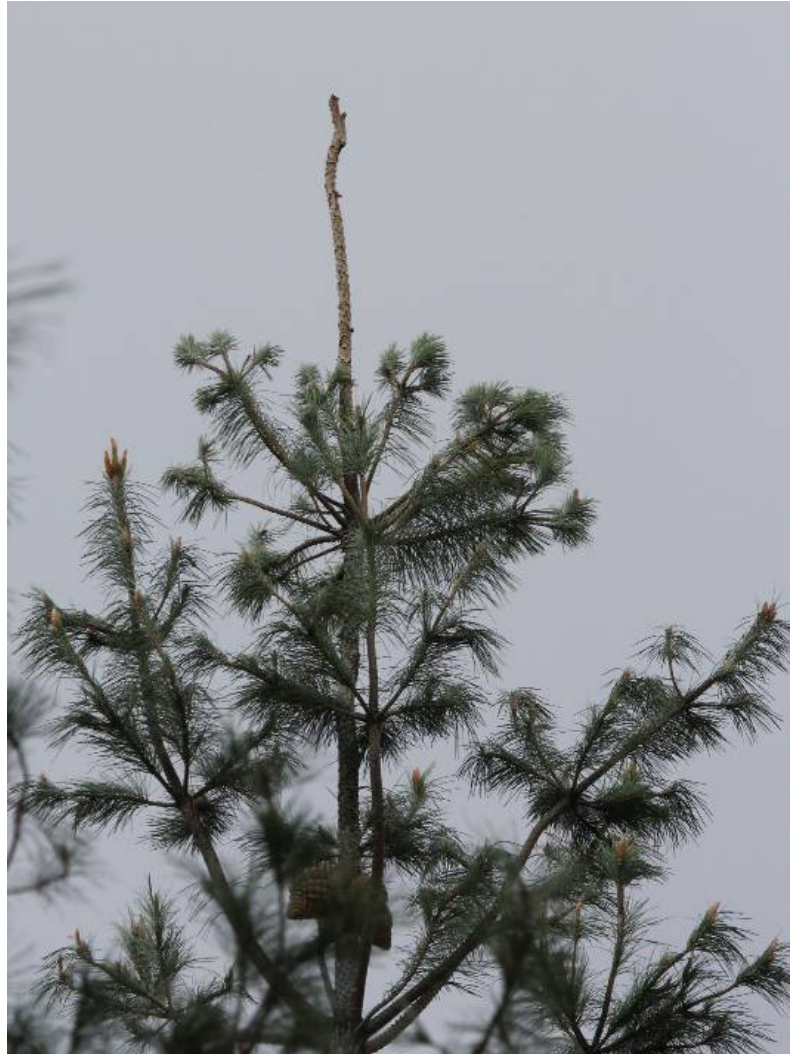

Figura 2. Defoliación apical en Pinus radiata causada por Pissodes castaneus.

Leader defoliation caused by Pissodes castaneus in Pinus radiata.

daño en las forestaciones ubicadas más al norte y disminuyendo los niveles de ataque hacia el sur. No se encontró una asociación entre la ubicación longitudinal y el grado de ataque $(r=0,11 ; P=0,55)$.

Se observó una variación en el porcentaje de infestación en función de la especie hospedera. La especie Pinus contorta var. murrayana resultó la más afectada y en orden de importancia le siguieron Pinus radiata y Pinus ponderosa (figura 3). La plaga afectó el $19 \%$ de los individuos de Pinus contorta var. murrayana muestreados, el 11,3\% de Pinus radiata y sólo el $3 \%$ de los individuos de Pinus ponderosa. Por otra parte, el mayor porcentaje de mortalidad correspondió a la especie Pinus contorta var. murrayana (76 \%) seguido por Pinus ponderosa (47,5\%) y Pinus radiata $(17 \%)$. La mortalidad de los árboles atacados por $P$. castaneus estuvo generalmente asociada con S. noctilio, sólo en plantaciones jóvenes de Pinus ponderosa fue registrado Pissodes castaneus como el único agente de daño, ocasionando la muerte de las plantas por anillamiento.

No existió una correlación entre el porcentaje de infestación y la edad de las plantaciones $(\mathrm{r}=0,12 ; P=0,47$ ). Tampoco hubo asociación entre el nivel de ataque y las variables dasométricas medidas $(\mathrm{DCM}, \mathrm{r}=0,148 ; P=0,398$; altura dominante, $\mathrm{r}=0,102 ; P=0,560$; IDR, $\mathrm{r}=0,149$; $P=0,394)$. 


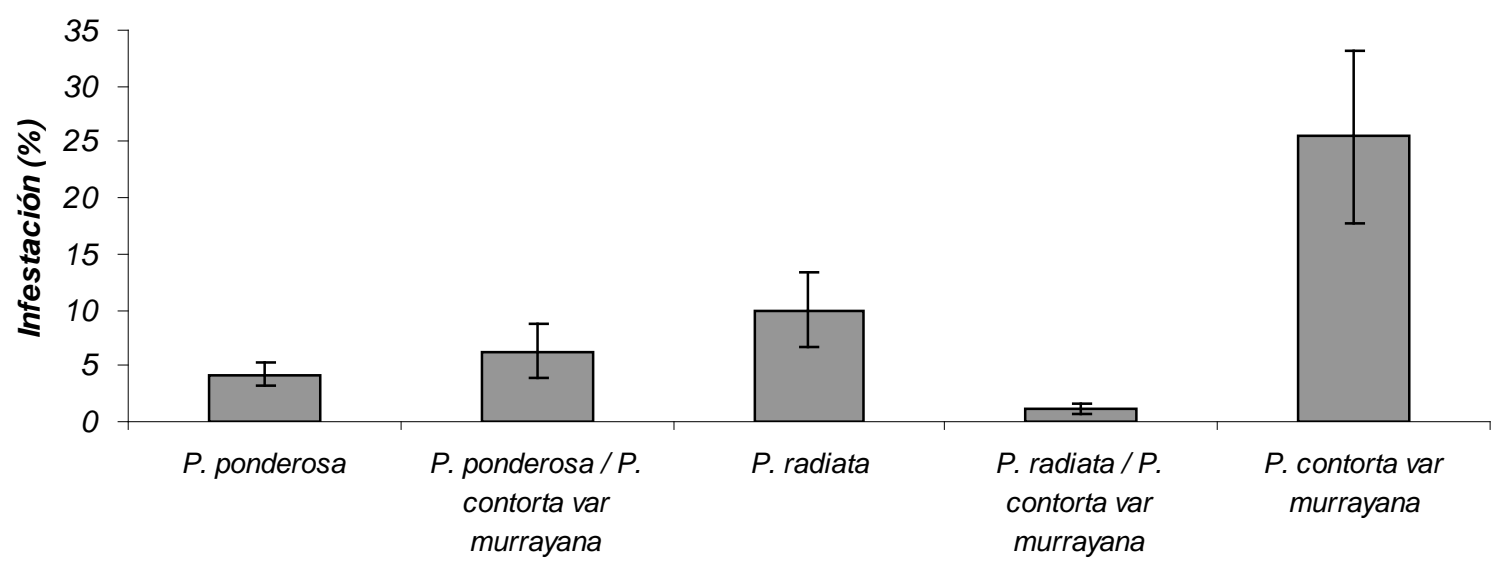

Plantaciones de Pinus spp.

Figura 3. Porcentaje de infestación por Pissodes castaneus en plantaciones de distintas especies de Pinus en la provincia de Chubut. Infestation level caused by Pissodes castaneus in different Pinus species plantations in Chubut province.

Si bien no se encontró una relación entre el nivel de ataque y la existencia de podas o raleos ( $r=0,075 ; P=0,669)$, una de las variables más relacionadas con la plaga fue el manejo de los residuos forestales. Existió una correlación positiva entre el grado de infestación por $P$. castaneus y la presencia de residuos forestales bajo el dosel $(r=0,59 ; P<0,001)$. En las forestaciones donde los restos de ramas y fustes, producto de las actividades de poda y raleo, fueron dejados en el suelo se observaron los mayores daños causados por la plaga. En los residuos forestales se registraron galerías, cámaras pupales con larvas, pupas y adultos vivos.

Relación con Sirex noctilio y otras especies de insectos. Existió una correlación positiva entre el grado de infestación por $P$. castaneus y S. noctilio $(\mathrm{r}=0,57 ; P<0,001)$. En la mayoría de las plantaciones muestreadas se registró la presencia de $S$. noctilio asociada con el gorgojo, observándose la coexistencia de cámaras pupales de P. castaneus y orificios de emergencia de la avispa a lo largo del fuste. No obstante, en estos sitios también se registraron plantas afectadas exclusivamente por la avispa y otras plantas afectadas exclusivamente por $P$. castaneus.

Pissodes castaneus fue registrado en plantas infestadas también por otras especies de insectos barrenadores, como Huequenia livida Germain (Coleoptera Cerambycidae), Rhyephenes maillei Gay et Solier (Coleoptera Curculionidae) e Hylurgus ligniperda F. (Coleoptera Scolytidae).

El análisis de componentes principales (ACP) muestra de manera conjunta la relación entre las distintas variables analizadas. El primer eje del ACP estuvo fuertemente asociado al porcentaje de afectación por S. noctilio (figura 4). Las plantaciones no afectadas por $P$. castaneus se ubicaron en valores negativos del eje 1 del ACP y valores positivos del eje 2, coincidiendo con sitios de mayor latitud, poco afectados por $S$. noctilio y sin presencia de residuos forestales. Por el contrario, aquellas parcelas más intensamente afectadas por $P$. castaneus, se ubicaron hacia valores positivos del eje 1 , coincidiendo con un mayor grado de afectación por $S$. noctilio y presencia de residuos forestales.

\section{DISCUSIÓN Y CONCLUSIONES}

La distribución regional de $P$. castaneus sugiere que el ingreso de la plaga se produjo en la zona sur de Río NegroNorte de Chubut y desde allí se dispersó. Sin embargo, pareciera que en Neuquén el ingreso de la especie ha sido más reciente que en Río Negro y Chubut, dada la escasa cantidad de forestaciones registradas con ataque en dicha provincia.

La distribución actual de la especie en la provincia de Chubut, de acuerdo a la sintomatología y daños observados en las forestaciones del noroeste, sugiere que $P$. castaneus estaría presente desde hace varios años antes de su detección circunstancial en el marco del programa de monitoreo de S. noctilio. Asimismo, sugiere junto con los niveles de infestación reportados, una antigüedad de invasión decreciente en sentido latitudinal, puesto que la sintomatología registrada hacia el límite sur de distribución de las plantaciones corresponde a etapas tempranas del ataque.

La asociación positiva entre el nivel de infestación por $P$. castaneus y el ataque de $S$. noctilio, se condice con la diagnosis del ataque que se reporta para las especies europeas de Pissodes. Estas son generalmente plagas secundarias, y los estallidos de $P$. castaneus están habitualmente asociados con plantas previamente debilitadas por la acción de otros insectos o patógenos (Day et al. 2004). La relación e interacción entre $P$. castaneus y las otras especies de insectos cohabitantes de la madera registradas en las plantas afectadas, es aún desconocida.

A pesar de lo expresado, existen observaciones de $P$. castaneus afectando árboles aparentemente sanos, en especial cuando alcanza altas densidades de población (Ku- 


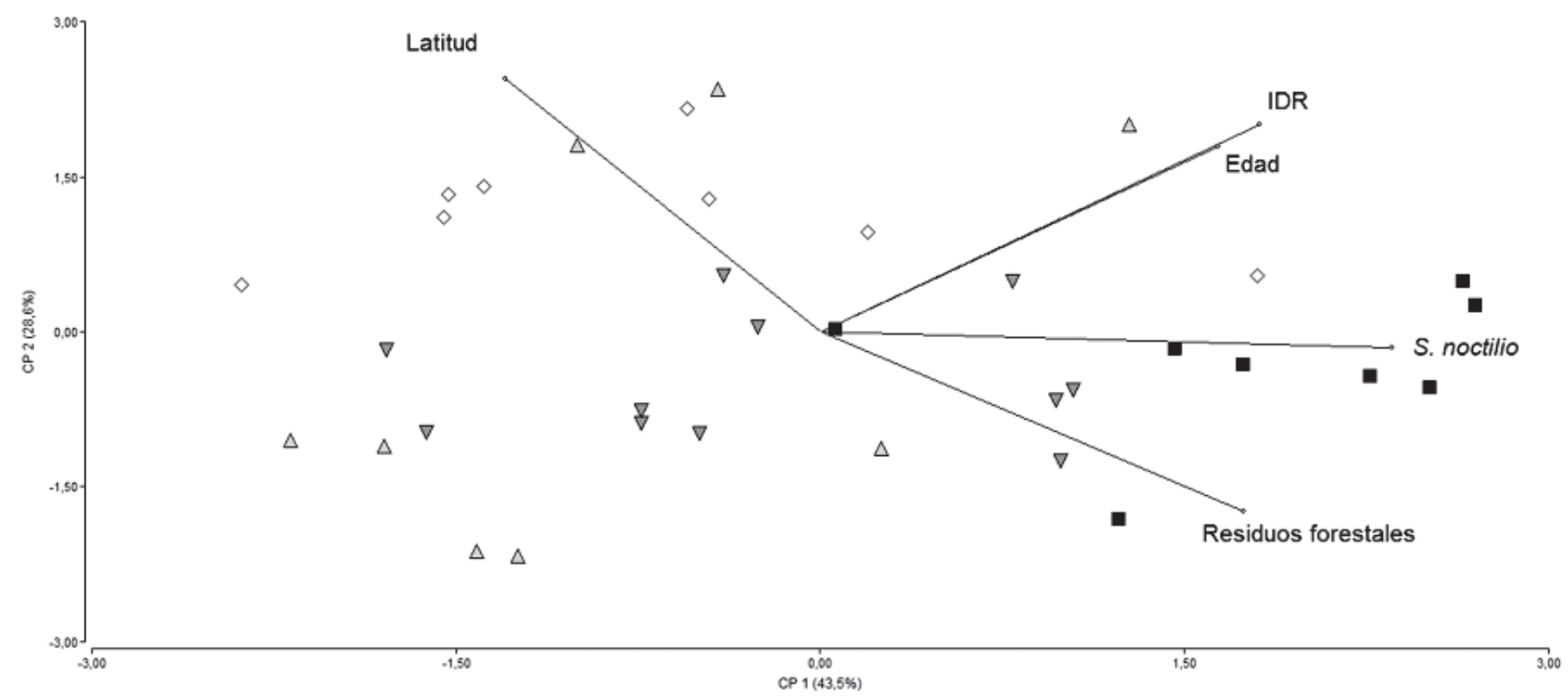

Figura 4. Análisis de componentes principales. Los símbolos indican distintos porcentajes de infestación por P. castaneus: 0 \% ( $\diamond)$, $0-4 \%(\mathbf{\Delta}), 4-10 \%,(\boldsymbol{\nabla}),>10 \%(\boldsymbol{\square})$.

Principal component analyses. References: $0 \%(\diamond), 0-4 \%(\mathbf{\Delta}), 4-10 \%,(\boldsymbol{\nabla}),>10 \%(\mathbf{\bullet})$.

dela 1974, Day et al. 2004). Esta situación, en la que las plantas afectadas por $P$. castaneus no padecen una condición de debilitamiento previo, también se ha observado en algunas de las plantaciones evaluadas en este estudio pero con bajos niveles de daño. Los estudios sobre la ocurrencia de la plaga en estos sitios deben profundizarse para comprender mejor la diagnosis del ataque.

Antecedentes en otros países, tales como Brasil y España, indican que el manejo silvicultural es una de las medidas más relevantes para el control de la especie (Romanyk y Cadahia 2003, Iede et al. 2004). Tanto el momento como la forma en que se realiza la poda pueden contribuir al incremento poblacional de $P$. castaneus. A través de las heridas o lesiones que puede ocasionar una poda inadecuada se liberan compuestos químicos, tales como monoterpenos, sesquiterpenos y etanol, que son los más relevantes en términos de la activación de los receptores neuronales en la especie (Bichão et al. 2003). Estos compuestos atraen al insecto, por lo que en Brasil se recomienda que la poda solo debe ser practicada en época invernal, fuera del período de oviposición (Iede et al. 2007). Esta misma prescripción también podría recomendarse en la región Andino Patagónica, a pesar que todavía no se conoce el ciclo de vida de la especie en el lugar y aspectos de su bioecología.

Si bien en este estudio no se encuentra una relación entre el nivel de ataque y la existencia de podas o raleos, es posible que el efecto positivo de estas intervenciones silviculturales haya sido enmascarado por los residuos que son dejados en el sitio. $\mathrm{Al}$ respecto, no es posible inferir si las podas fueron efectuadas cuando el insecto ya estaba presente en el rodal, o bien si el insecto arribó al lugar atraído por los desechos de las podas. Así, este estudio estableció que la falta de manejo de los residuos forestales se correlaciona positivamente con el grado de infestación, contribuyendo al aumento poblacional. Los residuos forestales que son dejados en el campo sin ningún tipo de tratamiento posterior, constituyen sitios de cría y refugio especialmente durante el invierno, por lo que son reservorios para el insecto (Cobos Suarez y Ruiz Urrestarazu 1990, Iede et al. 2007). Otros barrenadores del floema, como los escolítidos descortezadores, también poseen esta forma de vida. En Alemania los estallidos poblacionales de estos insectos suelen sucederse luego de la caída de gran cantidad de árboles a causa de tormentas de vientos muy fuertes. Estos árboles son dejados en el suelo y propician el incremento de las poblaciones (Schwenke 1974, Kaulfuß 2012).

Lamentablemente, la falta de manejo de los residuos es habitual en la región, fundamentalmente debido a razones logísticas y económicas. Sin embargo, esta práctica que durante años pudo sostenerse, debe ser revisada, dado el marco sanitario actual en el que se han reportado diferentes especies de descortezadores que hacen el mismo uso de los residuos que $P$. castaneus.

La continuidad de hospedadores en las forestaciones de pino en Patagonia, sumada a prácticas silviculturales inadecuadas o inexistentes facilitó la colonización y establecimiento de la especie en la región. Se sabe que los insectos fitófagos son favorecidos por la mayor disponibilidad de alimento y, en el caso de las especies introducidas, también por la falta de enemigos naturales que contribuyen a la ocurrencia de brotes de plagas. Una vez más, los hechos y la experiencia ganada con la avispa de los pinos 
en la región, destacan al manejo silvicultural como una condición indispensable para conferir la resistencia a los árboles, o bien al menos disminuir los niveles de ataque. De esta forma, es fundamental que las prácticas silviculturales se incorporen al manejo forestal integral desde un enfoque sanitario preventivo y no exclusivamente bajo la concepción de producción de madera.

Las especies que resultaron ser más susceptibles para $P$. castaneus son las mismas registradas para otras plagas en la región, como Rhyacionia buoliana Den et Schiff (Gomez 2004) y S. noctilio (Gomez 2008). En Pinus contorta var. murrayana el daño consiste principalmente en la muerte de la planta. Por otra parte, si bien Pinus ponderosa es aparentemente menos susceptible al ataque respecto de Pinus radiata, el insecto fue capaz de producir la muerte en plantas jóvenes.

Las características del ataque observado en la región, sumadas a la distribución que ha alcanzado la especie- especialmente en las provincias de Río Negro y Chubut- exigen que $P$. castaneus sea considerado en el manejo integral de las forestaciones, fundamentalmente en lo que se refiere al manejo de residuos forestales y raleos a desecho. La circulación de madera afectada es otro aspecto a considerar para evitar el establecimiento de la plaga en nuevas áreas. Por otro lado, el monitoreo de los niveles de daño es necesario para conocer el comportamiento de las poblaciones y evaluar el status sanitario de la especie.

\section{AGRADECIMIENTOS}

A los propietarios de los establecimientos forestales de Chubut, Río Negro y Neuquén, que nos permitieron el ingreso a sus plantaciones. A la Lic. Gabriela Tavella, Ingeniera Forestal Jessica Arre y a Iván Portscher por su asistencia en la tareas de campo.

\section{REFERENCIAS}

Assman E. 1970. The principles of forest yield study. Oxford. Pergamon Press. 506 p.

Bichão H, A Borg-Karlson, J Araújo, H Mustaparta. 2003. Identification of plant odours activating receptor neurones in the weevil Pissodes notatus F. (Coleoptera, Curculionidae). Journal of Comparative Physiology 189: 203-212.

Cobos Suarez J, M Ruiz Urrestarazu. 1990. Problemas fitosanitarios de la especie Pinus radiata D. Don en España, con especial referencia al País Vasco. Boletín Sanidad Vegetal Plagas 16: 37-53.

Daniel T, J Helms, F Backer. 1982. Principios de silvicultura. México D.F. McGraw-Hill. 492 p.

Day K, G Nordlander, M Kenis, G Halldorson. 2004. General biology and life cycles of bark weevils. In Lieutier F, K Day, A Battisti, J Gregoire, H Evans eds. Bark and Wood Boring Insects in Living Trees in Europe: a Synthesis. Netherlands. Springer. 339-349 p.

Fontana V, S Tiranti, D Zapiola. 2006. Pissodes castaneus De Geer "Gorgojo de la corteza del pino" un nuevo problema sanitario en las plantaciones de Pino en Norpatagonia. Revista Patagonia Forestal XII(3): 5-7.

Gomez C. 2004. Biología y control de la mariposa europea del brote del pino Rhyacionia buoliana Den. et Schiff. en la Patagonia, Argentina. Tesis doctoral. La Plata, Argentina. Facultad de Ciencias Naturales y Museo, Universidad Nacional de La Plata. 100 p.

Gomez C. 2008. Principales especies de insectos forestales en plantaciones de pino de Patagonia. Cuadernillo $n^{\circ} 3$. Serie Técnica: Manejo integrado de plagas forestales. Cambio Rural- Laboratorio de Ecología de Insectos. INTA EEA Bariloche.

Gomez C, M Hartel. 2010. El gorgojo de la corteza del pino, Pissodes castaneus. Cuadernillo $n^{\circ}$ 9. Serie Técnica: Manejo integrado de plagas forestales. Bariloche, Argentina. INTA EEA Bariloche. 14 p.

Gomez C, A Greslebin, M Rajchenberg. 2011. Plagas y enfermedades de Pinus sp. de la región Andino Patagónica de Argentina. Manual de campo. Buenos Aires, Argentina. Facultad de Ingeniería, Universidad Nacional de la Patagonia San Juan Bosco (UNPSJB). 85 p.

Gonda H. 2001. Pino ponderosa: productividad de las plantaciones en la Patagonia Andina. Esquel, Argentina. Centro de Investigación y Extensión Forestal Andino Patagónico. 8 p.

Iede E, W Reis Filho, S Penteado. 2004. Ocorrência de Pissodes castaneus (De Geer) (Coleoptera: Curculionidae) em Pinus, na Região Sul do Brasil. Brasil. Embrapa Florestas. 6 p. (Comunicado Técnico 114).

Iede E, W Reis Filho, S Zaleski, F Assis Marques, N Caldato. 2007. Monitoramento e controle de Pissodes castaneus em Pinus spp. Brasil. Embrapa Florestas. 8 p. (Circular Técnica 130).

Iede E, W Reis Filho, S Penteado, S Ribeiro Messa Zaleski. 2010. Pissodes castaneus (De Geer, 1775) (Coleoptera: Curculionidae) the bark pine weevil: a pest or a biological indicator? In McManus KA, KW Gottschalk eds. 21st U.S. Department of Agriculture Interagency Research Forum on Invasive Species, Annapolis, USA, January 2010. Proceedings. p. 95.

Kaulfuß S. 2012. Überwachung und Prognose von Schadinsekten. Consultado 5 de ago. 2013. Disponible en http:// www.waldwissen.net/waldwirtschaft/schaden/insekten/ fva_schadinsekten_is2/index_DE

Kudela M. 1974. Curculionidae, Pissodini. In Schwenke W ed. Die Forstschädlinge Europas. 2 Band. Hamburg, Germany. Paul Parey. 299-310 p.

Lanteri A, A Marvaldi, S Suárez. 2002. Gorgojos de la Argentina y sus plantas huéspedes. Tomo I: Apionidae y Curculionidae. San Miguel de Tucumán, Argentina. Special Publication 1. Sociedad Entomológica. 98 p.

MINAGRI (Ministerio de Agricultura, Ganadería y Pesca, AR). 2012. Elaboración de un mapa de plantaciones forestales de la República Argentina de actualización permanente. Área SIG e Inventario Forestal. Dirección de Producción Forestal. Ministerio de Agricultura Ganadería y Pesca. Presidencia de la Nación. Consultado 5 jul. 2012. Disponible en http://www.minagri.gob.ar/new/00/forestacion/inventario/ mapa\%20de\%20planta.html.

Morey C, J Porcile. 1992. Aspectos fitosanitarios del desarrollo forestal en Uruguay. Montevideo, Uruguay. Dirección Forestal, Ministerio de Ganadería Agricultura y Pesca. 30 p. 
O’Brien L. 1989. A catalog of the Coleoptera of America north of Mexico. Family Curculionidae, subfamily Pissodinae Bedell 1988. Agriculture Handbooks 529-143d. Tallahassee, Fl., USA. USDA Agricultural Research Service. 17 p.

Panzavolta T. 2007. Instar Determination for Pissodes castaneus (Coleoptera: Curculionidae) using head capsule widths and lengths. Environmental Entomology 36(5): 1054-1058.

Panzavolta T, R Tiberi. 2010. Observations on the life cycle of Pissodes castaneus in central Italy. Bulletin of Insectology 63(1): 45-50.

Ribeiro S. 2009. Pissodes castaneus (De Geer) (Coleoptera, Curculionidae): Bioecologia, feromônio sexual, variabilidade genética e aspectos do monitoramento e controle. Tese de
Pósgraduacao em Ciências Biológicas. Curitiba, Brasil. Universidade Federal do Paraná. 121 p.

Romanyk N, D Cadahia. 2003. Plagas de insectos en las masas forestales. Madrid, España. Mundi-Prensa. 336 p.

SAG (Servicio Agrícola y Ganadero, CL). 2013. Plagas cuarentenarias presentes. Consultado 5 de ago. 2013. Disponible en http://www.sag.cl/ambitos-de-accion/plagas-cuarentenarias-presentes

Schwenke W. 1974. Die Forstschädlinge Europas. 2 Band. Hamburg, Germany. Paul Parey. 488 p.

Villacide J, J Corley. 2012. Ecology of the woodwasp Sirex noctilio: Tackling the challenge of successful pest management. International Journal of Pest Management 58(3): 249-256.

Recibido: 06.06.13

Aceptado: 09.10.13 
